\title{
Sistemas de Informações em Saúde: Potencialidades e Dificuldades vivenciadas por profissionais/acadêmicos na prática assistencial
}

\author{
Health Information Systems: Potentials and Difficulties experienced by professionals/academics in \\ care practice
}

\section{Sistemas de información en salud: potencialidades y dificultades experimentadas por profesionales / académicos en la práctica asistencial \\ Vitor Duarte Celento ${ }^{1 *}$, Gabriel Porto Soares ${ }^{2}$, Denize Duarte Celento ${ }^{3}$, Thaiana Duarte Celento ${ }^{4}$, Gustavo Campos de Farias Kifer Moreira Ribeiro ${ }^{5}$, Yan Rodrigo de Almeida Machado ${ }^{6}$ \\ Como citar esse artigo. Celento, V. D; \\ Resumo}

Soares, G. P; Celento, D. D; Celento,

T. D; Ribeiro, G. C. F. K. M; Machado,

Y. R. A. Experiência emocional de enfermeiros atuantes na linha de frente durante a pandemia de Covid-19. Revista Pró-UniverSUS. 2021 Jul./Dez.; 12 (2): $07-16$

O presente estudo busca elucidar as dúvidas do cotidiano dos profissionais de saúde e dos acadêmicos da área de saúde, quanto aos sistemas de informações da saúde (SIS), e o acesso aos dados e informações confiáveis e pertinentes para o planejamento, implementação e tomada de decisão das suas atividades. Trata-se de uma pesquisa de natureza qualitativa, de caráter descritivoexploratório do estado da arte no período de 2010 a 2020. Os achados retratam o reconhecimento da evolução tecnológica e a importância das informações para a segurança e confiabilidade no planejamento da assistência à saúde. Porém, a realidade diversificada das regiões brasileiras ainda traz limitações. $\mathrm{O}$ aprimoramento é imprescindível e o processo é contínuo no tratamento dos dados locais, que deve levar em consideração o princípio da equidade no momento de elaborar novas políticas como a da informatização dos prontuários dos pacientes.

Palavras-chave: Sistema de Informação; Assistência à Saúde; Qualidade.

\begin{abstract}
This study seeks to elucidate the daily doubts of health professionals and academics in the health area, regarding health information systems (SIS), and access to reliable and relevant data and information for the planning, implementation and taking of decision of its activities. This is a qualitative, descriptive-exploratory research of the state of the art in the period from 2010 to 2020. The findings portray the recognition of technological evolution and the importance of information for safety and reliability in health care planning. However, the diversified reality of the Brazilian regions still has its limitations. Improvement is essential and the process is continuous in the treatment of local data, which must take into account the principle of equity when developing new policies such as the computerization of patient records.

Keywords: Information System; Health Care; Quality.
\end{abstract}

\section{Resumen}

Este estudio busca dilucidar las dudas cotidianas de los profesionales de la salud y académicos del área de la salud, sobre los sistemas de información en salud (SIS), y el acceso a datos e información confiables y relevantes para la planificación, implementación y toma de decisión de sus actividades. Se trata de una investigación cualitativa, descriptiva-exploratoria del estado del arte en el período de 2010 a 2020. Los hallazgos retratan el reconocimiento de la evolución tecnológica y la importancia de la información para la seguridad y confiabilidad en la planificación de la salud. Sin embargo, la realidad diversificada de las regiones brasileñas todavía tiene sus limitaciones. La mejora es fundamental y el proceso es continuo en el tratamiento de los datos locales, que debe tener en cuenta el principio de equidad a la hora de desarrollar nuevas políticas como la informatización de las historias clínicas.

Palabras clave: Sistema de Información; Atención a la Salud; Calidad.

Afiliação dos autores:

${ }^{1 *}$ Graduando de Medicina, Universidade de Vassouras. Vassouras, RJ, Brasil. e-mail: vitorduartecelento@gmail.com. ORCID: https://orcid.org/0000-0001-7698-4083.

${ }^{2}$ Médico/Cardiologista/ Doutorado em Cardiologia UFRJ. Professor do Curso de Medicina da Universidade de Vassouras, Vassouras, RJ, Brasil. e-mail: gp.soares@yahoo.com.br. ORCID: https://orcid.org/0000-0002-7058-799X.

${ }_{3}^{3}$ Administradora/Mestre em Ciências do Cuidado em Saúde. Professora do Curso de Enfermagem da Universidade de Vassouras, Vassouras, RJ, Brasil. e-mail: denize.celento@, hotmail.com. ORCID: https://orcid.org/0000-0002-1829-759X.

${ }^{4}$ Médica/Anestesista/ Mestranda do Mestrado Interinstitucional da Universidade de Vassouras / Médica do Hospital Universitário de Vassouras, Vassouras, RJ, Brasil. e-mail: thaianacelento@hotmail.com. ORCID: https://orcid.org/0000-0003-2456-9085.

${ }^{5}$ Graduando de Medicina/ Universidade de Vassouras, Vassouras, RJ, Brasil. e-mail: gugakifer@hotmail.com. ORCID: https://orcid.org/0000-0002-6159-9569.

${ }^{6}$ Graduando de Medicina/ Universidade de Vassouras, Vassouras, RJ, Brasil. e-mail: yan_06_@hotmail.com. ORCID: https://orcid.org/0000-0002-8042-4537.

*Email de correspondencia: vitorduartecelento@gmail.com

Recebido em: 13/07/21. Aceito em: 26/10/21. 


\section{Introdução}

Nas últimas décadas nos deparamos por diversas vezes com os conceitos: "informação", "qualidade"2 e "decisão"3 no nosso cotidiano, nas mais diversas atividades laborais e de formação profissional.

Emum mundo em constante evolução tecnológica, torna-se importante que as instituições em qualquer área do saber, sejam elas, públicas ou privadas, adaptem-se às novas perspectivas de trabalho. Essa exigência se converte para a criação e disseminação de informações, com rapidez; transmissão e acesso do conhecimento.

A informação é instrumento essencial à tomada de decisão. $\mathrm{Na}$ perspectiva da saúde, representa imprescindível ferramenta à vigilância em saúde. Com informações fragmentadas existirá o comprometimento no processo decisório, que permeia e orienta na implantação e na avaliação dos modelos de atenção à saúde. Oportunidade, atualidade, disponibilidade e cobertura são características que determinam a qualidade da informação, fundamentais para que todo o Sistema de Vigilância Epidemiológica apresente bom desempenho. ${ }^{4}$

Na área de saúde, nos últimos anos, diversos autores vêm destacando a importância do Sistema de Informação em Saúde (SIS) e o papel deste para as atividades de planejamento, execução, controle e avaliação da política de saúde, tanto em seus aspectos técnicos quanto gerenciais.

A motivação pelo presente tema do estudo surgiu em perspectiva familiar, com gestores, médicos e acadêmicos de medicina, diante das experiências nos ambientes de trabalho e na formação do profissional da saúde.

Emergem no cotidiano questionamentos de onde encontrar as informações e indicadores de saúde, capazes de atender as necessidades até então negligenciadas. Questões como: "Quais bases de dados? Onde e em qual tempo são registrados os dados oriundos das atividades? Questões que surgem e acabam por interferir no aumento da capacidade de intervir nos "nós" críticos, e nos problemas enfrentados diariamente.

O presente estudo tem como objetivo principal compreender as potencialidades dos Sistemas de Informação em Saúde - SIS e aponta como objetivos específicos demonstrar os principais Sistemas de Informações em Saúde, descrever a atuação/ interferência dos profissionais de saúde na alimentação dos Sistemas de Informações em Saúde e analisar o uso dos Sistemas de Informação em Saúde na formação desses profissionais.

Genericamente, o conceito de informação está intimamente ligado às noções de restrição, comunicação, controle, dados, forma, instrução, conhecimento, significado, estímulo, padrão, percepção e representação de conhecimento.

Os Sistemas de Informação em Saúde (SIS) devem incluir todos os dados necessários aos profissionais de saúde e utilizadores dos sistemas, com o objetivo de desenvolver e proteger a saúde das populações. Segundo a Organização Mundial de Saúde, o investimento nestes sistemas de informação possui vários benefícios e auxílio ao processo de tomada de decisão, no controle e detecção de problemas de saúde endêmicos, na monitorização de progressos e metas préestabelecidos e na promoção da equidade e da qualidade dos serviços. ${ }^{5}$

A Avaliação da qualidade em Saúde produz informações quanto à adequação, efeitos e custos associados ao uso de tecnologias, programas ou serviços de tecnologias e programas de saúde. Assim, pode subsidiar a tomada de decisão em relação às práticas ou serviços de saúde e ao estabelecimento de políticas concernentes ao setor. ${ }^{2}$

A busca por qualidade pelos serviços de atenção à saúde é uma necessidade técnica e social ${ }^{6}$, e a adoção de um sistema de gestão da qualidade é uma decisão estratégica das organizações ${ }^{7}$. Estas atividades precisam fazer parte do dia a dia das organizações prestadoras de serviços à saúde. Ainda, se o atrativo para o convênio com os serviços de saúde desviar-se de uma pura base de preços para uma combinação de preços, qualidade de serviço e de atendimento. A questão da qualidade tornar-se-á fundamental para a sobrevivência de muitas organizações. ${ }^{6}$

Todos têm pressupostos e defesas sobre a qualidade, mas falta a disciplina da experiência administrativa, a incorporação da metodologia da qualidade no plano estratégico e nas atividades diárias, a educação e o treinamento da organização inteira ${ }^{8}$.

Tomada de decisão é um processo cognitivo que resulta na seleção de uma opção entre várias alternativas. É amplamente utilizada para incluir preferência, inferência, classificação e julgamento, quer consciente ou inconsciente. Existem duas principais teorias de tomada de decisão, teorias racionais e teorias não racionais, variando entre si em um sem número de dimensões. ${ }^{3}$

$\mathrm{O}$ conceito de qualidade aplicado a cuidados à saúde é, na prática, abordado em termos de um conjunto de atributos desejáveis. Um estudo ${ }^{2}$ propõe sete atributos, por ele chamados de pilares da qualidade: eficácia, efetividade, eficiência, otimização, aceitabilidade, legitimidade e equidade. Desses atributos, três apresentam interesse especial para a rotina de trabalho nos laboratórios de controle de qualidade em saúde: eficácia, efetividade e eficiência.

Assim, torna-se importante, no processo de tomada de decisão, conhecer e determinar a origem das informações e que elas sejam relevantes, isto é, aquelas realmente necessárias ao processo decisório. Essas 
informações devem estar oportunamente disponíveis, o que implica a possibilidade de que sejam acessíveis, ou recuperáveis, quando necessário, dentro de um tempo de resposta ideal para subsidiar uma determinada decisão. À decisão vinculam-se duas dimensões que se referem à execução da ação e à avaliação da ação. $\mathrm{O}$ processo de execução implica o acompanhamento, o que requer sistemas informativos, dados corretos, para garantir a obtenção dos resultados desejados. ${ }^{9}$

A definição de Sistemas de Informação pode partir de duas diferentes abordagens. A primeira baseiase na abordagem sistêmica e gerencial da informação, cujo objetivo do Sistema de Informação é integrar os diferentes setores da organização, permitindo satisfazer tanto necessidades globais, quanto específicas da mesma.

A segunda abordagem relaciona sistemas de informação utilizando apenas o uso da informática. Um "Sistema de Informação (SI) é um sistema que cria um ambiente integrado e consistente, capaz de fornecer as informações necessárias à todos os usuários" ${ }^{10}$ ou ainda, é um sistema integrado homem-máquina que fornece informações de suporte à operações, gerenciamento, análise e funções de tomada de decisões em uma organização. ${ }^{11}$

O elemento principal dos sistemas de informação é a própria informação e são essas informações que auxiliarão na tomada de decisões. Mas, toda informação deve ter uma origem. Ela provém da interação entre tecnologias, metodologias e pessoas que atuam em conjunto em prol de alcançarem os objetivos da organização.

Corrobora O'Brien ao afirmar que todos os sistemas de informação utilizam recursos humanos, de hardware, software, dados e rede para executar atividades de entrada, processamento, saída, armazenamento e controle que transformam recursos de dados em produtos de informação. ${ }^{12}$

Para o Sistema Único de Saúde - SUS, os principais sistemas de informações de níveis populacionais são, de mortalidade (SIM), de nascimento (SINASC), ambulatorial (SIA-SUS), de internações hospitalares (SIH), de notificações de doenças (SINAN), de atenção básica (SIAB).

O Departamento de Informática do Sistema Único de Saúde (DATASUS) surgiu em 1991 com a criação da Fundação Nacional de Saúde (Funasa). $\mathrm{Na}$ época, a Fundação passou a exercer a função de controle e processamento das contas referentes à saúde que antes era da Empresa de Tecnologia e Informações da Previdência Social (DATAPREV). Foi então formalizada a criação e as competências do DATASUS, que tem como responsabilidade prover os órgãos do SUS de sistemas de informação e suporte de informática, necessários ao processo de planejamento, operação e controle.
Nessas 3 décadas de atuação, o DATASUS já desenvolveu mais de 200 sistemas que auxiliam diretamente o Ministério da Saúde no processo de construção e fortalecimento do SUS. Atualmente, o Departamento é um grande provedor de soluções de software para as secretarias estaduais e municipais de saúde, sempre adaptando seus sistemas às necessidades dos gestores e incorporando novas tecnologias, na medida em que a descentralização da gestão torna-se mais concreta.

O DATASUS dispõe de duas salas-cofre, uma em Brasília e outra no Rio de Janeiro, nas quais são mantidos os servidores de rede que hospedam a maioria dos sistemas do Ministério da Saúde. A estrutura de armazenamento de dados (STORAGE) do Departamento tem condições de armazenar informações sobre saúde de toda população brasileira. Além disso, disponibiliza links espalhados em várias cidades brasileiras com conexões com todos os Núcleos Estaduais do Ministério da Saúde, Funasa, Agência Nacional de Vigilância Sanitária (ANVISA), Casa do Índio e com as 27 secretarias estaduais de saúde. ${ }^{13}$

O e-SUS é uma das estratégias do Ministério da Saúde para desenvolver, reestruturar e garantir a integração desses sistemas, de modo a permitir um registro da situação de saúde individualizado por meio do Cartão Nacional de Saúde.

O nome, e-SUS, faz referência a um SUS eletrônico, cujo objetivo é sobretudo facilitar e contribuir com a organização do trabalho dos profissionais de saúde, elemento decisivo para a qualidade da atenção à saúde prestada à população.

O e-SUS Atenção Básica (e-SUS AB) é uma estratégia para reestruturar as informações da saúde na Atenção Básica em nível nacional. A qualificação da gestão da informação é fundamental para ampliar a qualidade no atendimento à população. A estratégia e-SUS faz referência ao processo de informatização qualificada do SUS em busca de um SUS eletrônico.

O sistema e-SUS AB foi desenvolvido para atender às necessidades de cuidado na Atenção Básica. Logo, o sistema poderá ser utilizado por profissionais das equipes de $\mathrm{AB}$, pelas equipes dos Núcleos de Apoio a Saúde da Família (NASF), do Consultório na Rua (CnR) e da Atenção Domiciliar (AD), oferecendo ainda dados para acompanhamento de programas como Saúde na Escola (PSE) e Academia da Saúde. A primeira versão do sistema apoia a gestão do processo de trabalho das equipes por meio da geração de relatórios, sendo que, a segunda versão contemplará várias ferramentas de apoio à gestão. ${ }^{14}$

\section{Materiais e Métodos}

Trata-se de uma pesquisa de natureza qualitativa, 
de caráter descritivo-exploratório do estado da arte. Os artigos foram levantados a partir dos bancos de dados BIREME / MEDLINE/ LILACS com busca bibliográfica, visando o aprofundamento do problema. Foram adotados os seguintes descritores: "sistema de informação", "assistência à saúde" e "qualidade", e foram considerados artigos publicados nos últimos 10 anos analisados criteriosamente.

Vistos e selecionados os seguintes critérios de inclusão: artigos publicados entre os anos de 2010 a 2020, dispostos nos idiomas inglês, português e/ou espanhol, cujos os textos se apresentassem na íntegra. Foram encontrados um total de 64 artigos, destes, apenas 22 artigos foram tratados por terem relação direta ao estudo.

\section{Resultados e Discussão}

Considerando que a temática é de suma importância para a equipe de saúde em todos os níveis de atenção e todos os profissionais que nelas interagem. A partir da análise dos artigos selecionados, foram elaboradas 3 categorias principais, as quais são discorridas a seguir:

\section{As dificuldades na Atenção Primária à Saúde se expressam em adequação nos diversos serviços}

Quanto a implementação e evolução da informatização dos processos nos diversos serviços que refletem o atendimento ambulatorial, nota-se a pesquisa em unidades básicas de Londrina (PR), com adoção três fontes de informação da Atenção Básica (AB) para o monitoramento no sistema (Cadastramento e AcompanhamentodeHipertensoseDiabéticos(Hiperdia), Sistema de Informação da Atenção Básica (SIAB) e fichas de aprazamento), que demonstrou um grande número de informações não registradas simultaneamente, o que pode dificultar a adoção de ações de promoção da saúde e de monitoramento desse agravo. ${ }^{15}$

Também foram encontradas incongruências entre o sistema e registro local no sistema SIS Pré-natal em um município paulista, que estavam relacionadas ao preenchimento inadequado das fichas de atendimento, além do controle escasso dos prontuários e agendamento nos estabelecimentos. ${ }^{16}$

Estudo voltado para os Centros de Informação e Assistência Toxicológica (CIAT), uma das principais fontes para notificação e registro de casos, consolidados

Tabela 1. Artigos encontrados e selecionados para a revisão.

\begin{tabular}{|c|c|c|c|c|}
\hline Título do artigo & Autores & Ano & Tipo Publicação & Revista \\
\hline $\begin{array}{l}\text { Utilização do e-SUS AB e fatores } \\
\text { associados ao registro de procedimentos } \\
\text { e consultas da atenção básica nos } \\
\text { municípios brasileiros / e-SUS AB }\end{array}$ & $\begin{array}{l}\text { Thum MA. } \\
\text { Baldisserotto J. } \\
\text { Celeste RK. }\end{array}$ & 2019 & Estudo ecológico & $\begin{array}{l}\text { Cad. Saúde Pública } \\
\text { (Online) ; 35(2): } \\
\text { e00029418, 2019. }\end{array}$ \\
\hline $\begin{array}{l}\text { Avaliação do uso de sistemas de } \\
\text { prontuário eletrônico nas unidades de } \\
\text { terapia intensiva brasileiras }\end{array}$ & $\begin{array}{l}\text { Colleti JJ. } \\
\text { Andrade AB. } \\
\text { Carvalho WB. }\end{array}$ & 2018 & Estudo descritivo & $\begin{array}{l}\text { Rev. bras. ter. intensiva } \\
\text { 30(3): } 338-346, \text { jul.-set. } \\
2018 .\end{array}$ \\
\hline $\begin{array}{l}\text { Informatização da atenção básica a } \\
\text { saúde: avanços e desafios }\end{array}$ & $\begin{array}{l}\text { Cavalcante RB. } \\
\text { Vasconcelos } \\
\text { DD. Gontijo TL. } \\
\text { Guimarães EAA. } \\
\text { Machado RM. } \\
\text { Miranda OVC. }\end{array}$ & 2018 & $\begin{array}{l}\text { Estudo descritivo e } \\
\text { análise de conteúdo }\end{array}$ & $\begin{array}{c}\text { Cogitare enferm ; 23(3): } \\
\text { e54297, 2018. }\end{array}$ \\
\hline $\begin{array}{l}\text { Qualidade dos dados de assistência pré- } \\
\text { natal na Atenção Básica em prontuário } \\
\text { eletrônico e relação com apoio matricial, } \\
\text { Vitória, Espírito Santo, 2013-2014: corte } \\
\text { transversal }\end{array}$ & $\begin{array}{l}\text { Ruschi GEC. } \\
\text { Antônio FF. } \\
\text { Zandonade E. } \\
\text { Miranda AE. }\end{array}$ & 2017 & $\begin{array}{l}\text { Estudo exploratório } \\
\text { descritivo }\end{array}$ & $\begin{array}{l}\text { Rev. bras. med. fam. } \\
\text { comunidade; 12(39): 1-13, } \\
\text { jan.-dez. } 2017\end{array}$ \\
\hline
\end{tabular}


Tabela 1 (cont.). Artigos encontrados e selecionados para a revisão.

Título do artigo
Coordenação da assistência à
tuberculose: registro de dados e
a implementação de um sistema
informatizado
Aceitação e uso de tecnologias
móveis de informação pelos agentes
comunitários de saúde de Sapeaçu

Prontuário Eletrônico e Gerenciamento de caso em Ambulatório de Psiquiatria

Uso do prontuário eletrônico na assistência pré-natal às portadoras de diabetes

Avaliação do impacto da informatização dos registros sobre o parto na produção do conhecimento científico, em um hospital universitário

Cobertura do Sistema de Informações sobre Nascidos Vivos e potenciais fontes de informação em municípios de pequeno porte em Minas Gerais, Brasil

Atividades registradas por profissionais de saúde da família no sistema de informação da atenção básica

Bases de dados orçamentários e qualidade da informação: uma avaliação do Finanças do Brasil (Finbra) e do sistema de informações sobre orçamentos públicos em saúde (Siops)

Indicador de qualidade assistencial úlcera por pressão: análise de prontuário e de notificação de incidente

Atualização dos dados nos sistemas de informação em saúde

$\begin{array}{ccc}\text { Autores } & \text { Ano } & \text { Tipo Publicação } \\ \text { Orfão NH. } & & \begin{array}{c}\text { Estudo descritivo } \\ \text { com base de fontes } \\ \text { Crepaldi NY. }\end{array} \\ \text { Brunello MEF. } & & \\ \text { Andrade RLP. } & 2017 & \\ \text { Monroe AA. } & & \\ \text { Rufino NA. } & & \text { Estudo exploratório } \\ \text { Antonio VTS. } & & \text { descritivo } \\ \text { Daltro EFMA. } & & \\ \text { Barbosa DSJ. } & 2017 & \\ \text { Machado APR. } & & \\ \text { Santos EMB. } & & \text { Estudo de caso } \\ \text { Raul MM. } & & \text { com pesquisa } \\ \text { Silveira ASA. } & & \text { documental }\end{array}$

Feitosa ACR.

2016

Ávila AN.

Carzola LCM.

Gaspar JS.

Reis ZSN.

Girodo AM.

Campos D.

Bittencourt SDA.

Szward WCL.

França EB.

Cavalcante RB.

Gontijo TL.

Guimarães EAA.

Oliveira VC.

Martins JRT.

Medeiros KR.

Albuquerque PC.

Diniz GTN.

Alencar FL.

Tavares RAW.

Santos CT.

Oliveira MC

Pereira AGS.

Suzuki LM.

Estudo exploratório descritivo

Estudo

Exploratório descritivo

Lucena AF.

Jesus MCP.

Santos SMR.

Goulart TP.

Martins NA.

2012

Estudo ecológico exploratório
Ciênc. saúde coletiva ,

22(6): 1969-1977, jun. 2017

Rev. baiana saúde

pública ; 41(2): https://

doi.org/10.22278/2318-

2660.2017.v41.n2.a1333, abr. 2017.

J. health inform ; 8(3): [83-86], jul.-set. 2016.

Rev. bras. ginecol. obstet 38(1): 9-19, jan. 2016

J. health inform ; $8($ supl.I): 187-193, 2016

Rev. bras. saúde matern. infant ; 15(3): 317-324, jul.-set. 2015

Rev. eletrônica enferm ; 16(4): 736-743, 2014

Rev. adm. pública ; 48(5):

1113-1133, 2014.

Peres RB.

Coelho VS.
Rev. enferm. UERJ ; 20(2,n.esp): 795-801, dez. 2012 
Tabela 1. Artigos encontrados e selecionados para a revisão.

\begin{tabular}{ccccc}
\hline Título do artigo & Autores & Ano & Tipo Publicação & Revista \\
\hline
\end{tabular}

Implantação do Plano Educacional no prontuário eletrônico para pacientes e familiares

Política de saúde do homem

Práticas integrativas e complementares: oferta e produção de atendimentos no SUS e em municípios selecionados

Cobertura da Comunicação de Informação Hospitalar e Ambulatorial (CIHA) utilizando os partos registrados no Sistema de Informações sobre Nascidos Vivos, Brasil, 2006 a 2009

O prontuário eletrônico do paciente no sistema de saúde brasileiro: uma realidade para os médicos?: [revisão]

\begin{abstract}
Sistema nacional de informações tóxico-farmacológicas: o desafio da padronização dos dados
\end{abstract}

Sistema de Informação Pré-Natal: análise crítica de registros em um município paulista

Análise de três fontes de informação da atenção básica para o monitoramento da hipertensão arterial
Ruiz LD.

Laurino PS.

Roma M.

Schwarz E.

Gomes R.

Couto MT.

Moura EC.

Carvalho SA.

Silva SFC.

Sousa IMC.

Bodstein RCA.

Tesser CD.

Santos FAS.

Hortale VA.

Pinheiro RS.

Silva JRN.

Lima CRA.

Coeli CM.

Patrício CM.

Machiavelli JL.

Maia MM.

Navaes MA.

Santana RAL.

Bochner R.

Guimaraes MCS.

2011

Estudo exploratório

descritivo

Moimaz SAS

Garbin CAS.

Garbin AJI.

Zina LG.

Yaris SD.

Francisco KMS.

Girotto E.

Andrade SM.

Cabrera MAS.

2012

Relato de

experiência

2012

Estudo exploratório descritivo

Estudo exploratório descritivo

2011

Revisão de

literatura

2010

Estudo exploratório descritivo 2010

Estudo exploratório descritivo
J. health inform ;

4(esp.,pt.1)dez. 2012

Cad. saúde pública ; 28(11): 2143-2154, nov. 2012

Rev. saúde pública ; 46(supl.1): 108-116, Dez. 2012.

Sci. med ; 21(3)jul.- set. 2011.

Ciênc. saúde coletiva ; 16(supl.1): 1191-1200, 2011

Rev. bras. enferm ; 63(3): 385-390, maio-jun. 2010

Epidemiol. serv. saúde ; pelo Sistema Nacional de Informações TóxicoFarmacológicas (SINITOX). Uma vez que, a incidência de intoxicações e envenenamentos constitui um grave problema de saúde pública. O relacionamento das bases de dados é importante para estimar a magnitude da ocorrência dos óbitos. Os resultados apontaram para o baixo grau de padronização dos dados que chegam ao SINITOX, o que impacta, qualitativamente, as informações geradas por este sistema. ${ }^{17}$

Em análise à Política Nacional de Práticas Integrativas e Complementares (PICS), que incentiva a inserção e fortalecimento na atenção primária, um estudo buscou identificar a oferta dos serviços e de práticas integrativas e complementares no SUS e a produção de atendimentos entre os anos 2000 e 2011, analisando as informações disponíveis nos bancos de dados nacionais "vis-à-vis" os dados da atenção primária nos municípios de Campinas (SP), Florianópolis (SC) e Recife (PE). A análise permitiu identificar um descompasso entre o que se registra nos sistemas de informação e o que se pratica nos municípios. ${ }^{18}$

Estudo que retrata a discussão de articulação 
entre sistemas de informações epidemiológicas, produção científica e políticas de saúde de assistência à saúde do homem, considerou como necessidade, ampliação de utilização dos dados dos sistemas de informações epidemiológicas do Ministério da Saúde e que procedam à incorporação crítica da perspectiva relacional de gênero. ${ }^{19}$

Revela-se também o registro dos dados manualmente como uma das principais dificuldades para a atualização do Sistema de Informação da Atenção Básica e o Sistema de Vigilância Alimentar e Nutricional. Conforme o resultado do estudo realizado com os dados de 37 municípios, da Zona da Mata de Minas Gerais, sendo a maioria com menos de 20 mil habitantes, as menores taxas de cobertura do Sistema de Informação da Atenção Básica - SIAB estão nas cidades mais populosas. Os digitadores salientam essa dificuldade e reconhecem a importância do consolidado de informações para o planejamento das políticas públicas de saúde e assistência ao usuário. ${ }^{20}$

Surge ainda, a necessidade de adaptar o SIAB de acordo com a sua proposta de implantação, de organizar e aprimorar a qualidade das informações para subsidiar o monitoramento, a avaliação e o planejamento, segundo as prioridades de saúde da população. Estudo de 2014, apresenta que as principais atividades registradas pelo conjunto das equipes de saúde da família de Minas Gerais são as individuais, como consultas e atendimentos, retratando a concepção do próprio sistema que prioriza na coleta as atividades individuais e focadas em procedimentos.

Tal estudo destacou que é preciso buscar o aprimoramento do SIAB além dos aspectos tecnológicos, enfatizando o seu fluxo informacional, os processos de trabalho que instrumentaliza, a realidade diversificada das regiões brasileiras e as demandas informacionais da equipe multidisciplinar. Apontam a necessidade de aprimoramento dos instrumentos de coleta para que a consolidação dos dados represente a diversidade de atividades realizadas no cotidiano das equipes de saúde da família. ${ }^{21}$

Visto a relevância da integração entre os diversos cenários da saúde, aborda-se o sistema de nascidos vivos (SINASC). Estudo indica problemas na qualidade da informação dos registros vitais em municípios de pequeno porte, relacionados principalmente a problemas na coleta e no fluxo das informações do SINASC nos municípios da mesorregião do Jequitinhonha, Minas Gerais.22

No segmento obstétrico e endocrinológico de gestantes que são acompanhadas no ambulatório de endocrinopatias na gestação na maternidade referência em gestação de alto risco na Bahia, em um período de 3 anos houve a validação positiva do Registro Eletrônico de Saúde (RES). A taxa de preenchimento de informações clínicas apresentou-se superior à do prontuário convencional. ${ }^{23}$

Com base na implantação do prontuário eletrônico no ambulatório de psiquiatria, em substituição ao manuscrito, em outubro de 2012. Concomitantemente, criado um sistema eletrônico de integração das informações dos principais dados dos pacientes em um relatório de gerenciamento de caso em tempo real. Houve uma melhor integração das informações dos pacientes, com maior segurança e confiabilidade. Apesar de surgirem algumas dificuldades de assistência técnica e complexidade do sistema. Os benefícios do prontuário eletrônico superaram as dificuldades, garantindo um melhor funcionamento do gerenciamento de caso. ${ }^{24}$

Também em experiência exitosa com agentes comunitários na Bahia, a inserção e uso de tecnologias móveis (tablets), para registro e transmissão de dados de informação. Apresentou resultado favorável, com superação de expectativas de desempenho. ${ }^{25}$

Outro estudo que aponta a satisfação na implantação da tecnologia, foi na coordenação de assistência à tuberculose, com o sistema informatizado para registro dos dados da assistência de tuberculose em Ribeirão Preto (SP). ${ }^{26}$

A implantação do sistema possibilitou a melhora no registro de algumas variáveis, ainda que, em outras fontes de registro foram mantidas. Neste sentido, destaca-se a importância de implantar o SISTB em outros cenários para compreender de que forma ele contribui para o adequado manejo clínico, vigilância, avaliação e planejamento em saúde, bem como na coordenação da informação e gestão da atenção, a partir de diferentes experiências e organização da rede de atenção à saúde. ${ }^{26}$

A avaliação da qualidade dos dados dos prontuários eletrônicos das gestantes na APS, realizado em Vitória - ES, no período de 1 de janeiro de 2013 a 31 de dezembro de $2014^{27}$. Concluiu o potencial do prontuário eletrônico como fonte de informação epidemiológica sobre a assistência pré-natal. Contudo, sua confiabilidade é prejudicada pela falta de integração dos dados com os demais níveis de atenção e sistemas de informação, e sua completude é deficiente em alguns aspectos. Houve ainda, discordâncias com o sistema de informação de Nascidos Vivos.

O trabalho contribuiu para reforçar a necessidade de treinamento e capacitações periódicas dos profissionais para um adequado manuseio do sistema de informação local, que sofre ajustes periodicamente, sem orientações prévias da gestão e do departamento de informática da Secretaria Municipal de Saúde. ${ }^{27}$

A análise à implantação da estratégia e-SUS Atenção Básica, na região oeste de $\mathrm{MG}$, mostrou fragilidades relacionadas à infraestrutura, deficiências nas capacitações e resistência dos profissionais. ${ }^{28}$ $\mathrm{O}$ processo de implantação apesar de sua evolução, apresentou fragilidades relacionadas aos responsáveis 
por acompanhar a implantação, por ter infraestrutura insuficiente nas UBS, deficiências nas capacitações, resistência dos profissionais e efeitos negativos sobre o trabalho.

É preciso reconhecer politicamente a importância da informatização como suporte na organização do trabalho na atenção básica e a gestão do cuidado sob a ótica dos profissionais, também se fazem necessárias. ${ }^{28}$

Ao avaliar o impacto do e-SUS AB nos municípios brasileiros no período de 2013 a 2015, quanto notificação de procedimentos e consultas no Sistema de Informação Ambulatorial do Sistema Único de Saúde (SIA/SUS), não foram observadas diferenças nas taxas de procedimentos ou consultas entre municípios de acordo com a fase de implantação e também não houve aumento ao longo do tempo. ${ }^{29}$

Relevante salientar que os municípios de grande porte, com mais de 100 mil habitantes, apresentaram uma taxa de procedimentos e consultas menores do que os demais. Esses municípios com maior número de habitantes, costumam ter maiores investimentos em serviços de média e alta complexidades e menores gastos com atenção primária em saúde. ${ }^{29}$

Revela-se também diferenças entre as regiões brasileiras, e paradoxalmente a Região Norte que registra as maiores taxas de consulta, também apresentou as menores taxas de procedimentos. Demonstraram ainda, heterogeneidades no registro de procedimentos ambulatoriais entre as regiões. Esses achados apresentaram como ponto forte, a inclusão de todos os municípios brasileiros, eliminando-se a possibilidade de viés de seleção. O estudo concluiu que outras análises devem ser aprofundadas quanto ao uso do sistema e suas implicações nos processos de trabalho das equipes para apoiar os gestores no uso e manutenção do sistema. ${ }^{29}$

\section{SIS no âmbito hospitalar - demonstração de impactos positivos}

O estudo e análise das vantagens e desvantagens do Prontuário Eletrônico do Paciente - PEP, demonstrou a importância da sua implementação nos serviços médico-hospitalares, os aspectos éticos envolvidos e os desafios para uso desse recurso pelos médicos, de modo a subsidiar a tomada de decisão sobre o uso ou não dos prontuários eletrônicos na prática médica.

Retratou que apesar das dificuldades relatadas, acredita-se que é de suma importância a utilização de sistemas de informação que incluam o prontuário eletrônico no âmbito do sistema de saúde brasileiro, a fim de identificar os usuários, facilitar a gestão dos serviços, a comunicação e o compartilhamento das informações em um país com dimensões continentais e imensa diversidade cultural. O PEP proporcionará mais qualidade ao atendimento e à gestão pública, com condições de superar os desafios para implantação e tornar-se uma prática comum na medicina. ${ }^{30}$

Em hospital privado no município de São Paulo, a elaboração e implantação de plano educacional, através de "workflow" integrado, para estimular o registro sistematizado de ações educativas no Prontuário Eletrônico do Paciente (PEP), pela equipe multiprofissional foi bem-sucedida, com vantagem de acesso remoto e simultâneo dos dados e compartilhamento de informações. ${ }^{31}$

Buscando ainda o impacto do uso de um sistema computadorizado de registro médico no número e qualidade de publicações científicas, em um hospital universitário, por parte dos professores dos departamentos de Ginecologia e obstetrícia, não foram encontradas evidências de que a informatização de parte dos registros médicos nesta maternidade tenha resultado em impacto imediato na produção científica publicada. Há ainda expressivos desafios para que o uso dos sistemas eletrônicos de informação na saúde possa beneficiar diretamente a disponibilidade e qualidade de dados de forma a fomentar diretamente o avanço da ciência médica em instituições acadêmicas de ensino e pesquisa. $^{32}$

Os achados ao examinar a prevalência de uso de sistemas de prontuários eletrônicos nas unidades de terapia intensiva brasileiras, e a percepção dos médicos intensivistas, em relação a contribuição dos sistemas de prontuário eletrônico para melhoria e qualidade na prática clínica, verificou a ampla utilização dos sistemas de PEP, a confiabilidade de maior qualidade e segurança em relação ao prontuário de papel. ${ }^{33}$

\section{O controle na área de financiamento da saúde}

Para acompanhamento da assistência prestada à população assistida com financiamento no SUS, foi instituída pelo Ministério da Saúde, em março de 1999, a obrigatoriedade do preenchimento da Comunicação de Internação Hospitalar $(\mathrm{CIH})$, para todos os estabelecimentos privados situados no território nacional.

A CIH passa, então, a ser instrumento obrigatório de informação dos eventos de internação realizados sem o financiamento do SUS, a serem encaminhadas pelos estabelecimentos de saúde às Secretarias Municipais/ Estaduais de Saúde, que têm a responsabilidade de transmissão ao Ministério da Saúde. Em outubro de 2005, é instituída, no âmbito da Agência Nacional de Saúde Suplementar(ANS), a CIH para acompanhamento da assistência prestada aos beneficiários de planos de saúde.

Um estudo mostrou a obrigatoriedade do preenchimento da Comunicação de Informação 
Hospitalar e Ambulatorial (CIHA) em todos os serviços de saúde do país, tomando como padrão os dados do Sistema de Informações sobre Nascidos Vivos (SINASC) do período de 2006 a 2009. ${ }^{34}$

A cobertura da CIHA foi baixa em quase todas as UF, menos de um quarto dos partos que foram registrados no SINASC, decrescendo desde $2006(24,4 \%)$ até 2009 (19,7\%). Mecanismos de supervisão da coleta e o uso da CIHA para apoiar a decisão são atividades importantes na sua constituição como um instrumento para planejamento e avaliação da assistência. ${ }^{34}$

No seguimento de finanças e orçamento público, destaca-se a avaliação dos sistemas de Finanças do Brasil (Finbra) e o Sistema de Informações sobre Orçamentos Públicos em Saúde (Siops) nas dimensões de qualidade de acessibilidade, oportunidade e clareza metodológica. Os sistemas apresentam semelhantes vantagens quanto à acessibilidade e oportunidade, mas o Siops revelou-se com melhor clareza metodológica. Havendo a necessidade de ampliar o conhecimento sobre essas importantes fontes de registro contábil dos entes subnacionais, com vista a seu melhoramento e aperfeiçoamento. ${ }^{35}$

\section{Considerações Finais}

O estudo retrata que os profissionais da saúde reconhecem a evolução tecnológica e a importância das informações para a segurança e confiabilidade no planejamento da assistência à saúde. Porém, a realidade diversificada das regiões brasileiras ainda traz as limitações apresentadas nos diversos artigos pesquisados.

Nota-se que o aprimoramento do processo de trabalho; o fluxo de informação; a instrumentalização dos dados e a completude das informações clínicas são essenciais para a eficácia do planejamento e gestão da saúde.

Apesar das pesquisas apontarem para a evolutiva adesão aos prontuários eletrônicos no âmbito hospitalar, o benefício é restrito somente àquela instituição de saúde. A dimensão capaz de atender a complexidade da saúde, requer aprimoramento nos segmentos da atenção coletiva e atenção hospitalar nos contextos de infraestrutura; coleta e registros de dados; capacitações; implantação e implementação dinâmica diante ao reconhecimento das prioridades da saúde da população.

Aponta-se para um processo contínuo no tratamento dos dados locais, e deve-se levar em consideração o princípio da equidade no momento de elaborar novas políticas como a da informatização dos prontuários dos pacientes. É importante salientar a preocupação que se deve ter para garantir a privacidade e confiabilidade dos dados contidos no prontuário eletrônico em busca da confiabilidade das informações do "SIS".

\section{Referências}

1. Portal da Educação. Conceito de Informação. Plataforma Portal da Educação. [Internet]. São Paulo, c2020 [acesso em 30 maio 2020]. Disponível em: https://www.portaleducacao.com.br/conteudo/artigos/informatica/ conceito-de-informacao/18895.

2. Donabedian, A. The Seven Pillars of Quality. Arch Pathol Lab Med, 1990. 1115-1119.p

3. Wikipédia: a enciclopédia livre. [Internet]. [São Francisco, CA: Fundação Wikipédia]; 2020. Tomada de Decisão. [acesso em 30 maio 2020]. Disponível em: https://pt.wikipedia.org/wiki/Tomada_de_decis\%C3\%A3o.

4. Brasil, Ministério da Saúde. Guia de vigilância epidemiológica .7. ed. - Brasília: 2009.

5. Brasil, Ministério da Saúde. Sistemas de Informação. [Internet]. Brasília: DF, c2020 [acesso em 06 Jun 2020]. Disponível em: http:// www.saude.gov.br/gestao-do-sus/programacao-regulacao-controle-efinanciamento-da-mac/sistemas-de-informacao-em-saude.

6. Quinto Neto A. Processo de acreditação: A busca da qualidade nas organizações de saúde. Porto Alegre: Dacasa: Palmarica; 2000. 136p.

7. Associação Brasileira de Normas Técnicas NBR ISO 9001. Sistemas de gestão da qualidade - requisitos. Rio de Janeiro, 2001

8. Berwick DM, Godfrey AB, Roessner J. Melhorando a qualidade dos serviços médicos, hospitalares e da saúde. Santos JCB, tradutor. São Paulo: MAKRON Books do Brasil; 1990.

9. Campos F E. Resolutividade - uma aproximação à avaliação qualitativa dos serviços de saúde. [dissertação]. Rio de Janeiro: Escola Nacional de Saúde Pública, Fiocruz; 1988. 264p.

10. Spinola M, Pessôa M. Tecnologia da Informação In: Gestão de Operações. 2a ed. São Paulo: Editora Edgard Blücher,1998. 97-104 p.

11. Schutzer E, Pereira AN. et al. Sistemas de informação. Gestão Agroindustial. Grupo de Estudos e Pesquisas Agroindustriais - GEPAI. São Paulo: Atlas, 1999. 147-168.

12. O’Brien JA. Sistemas de informação: e as decisões gerenciais na era da Internet. 2. ed. São Paulo: Saraiva 2004.

13. Brasil, Ministério da Saúde. Datasus. [Internet]. Brasília: DF. [acesso em 10 maio 2020]. Disponível em: http://datasus.saude.gov.br.

14. Brasil, Ministério da Saúde. Rol de Diretrizes, Objetivos, Metas e Indicadores 2013-2015 - Edição 2015 Datasus. [Internet]. Brasília: DF. [acesso em 10 maio 2020]. Disponível em: http://datasus.saude.gov.br/ projetos/50-e-sus.

15. Girotto E, de Andrade SM, Cabrera MASC. Análise de três fontes de informação da atenção básica para o monitoramento da hipertensão arterial. Epidemiologia e Serviços de Saúde. 2010. 19(2); 133-141.

16. Moimaz, S.A.S., et al. Sistema de Informação Pré-Natal: análise crítica de registros em um município paulista. Revista Brasileira de Enfermagem. 2010. 63; 385-390

17. de Santana, R.A.L., Bochner R., Guimarães M.C.S. Sistema nacional de informações tóxico-farmacológicas: o desafio da padronização dos dados. Ciência \& Saúde Coletiva. 2011. 16; 1191-1200.

18. de Sousa, Islândia M.C., et al. Práticas integrativas e complementares: oferta e produção de atendimentos no SUS e em municípios selecionados. Cadernos de Saúde Pública. 2012. 28; 2143-2154.

19. Schwarz, Eduardo, et al. Política de saúde do homem. Revista de Saúde Pública 46. 2012. 108-116.

20. de Jesus MCP, et al. Atualização dos dados nos sistemas de informação em saúde. Revista Enfermagem UERJ.2012. 20(6); 795-801.

21. Cavalcante RB, et al. Atividades registradas por profissionais de saúde da família no sistema de informação da atenção básica. Revista Eletrônica de 
Enfermagem. 2014. 16(4); 736-43.

22. Girodo AM, et al. Cobertura do Sistema de Informações sobre Nascidos Vivos e potenciais fontes de informação em municípios de pequeno porte em Minas Gerais, Brasil. Revista Brasileira de Saúde Materno Infantil. 2015. 15; 17-324.

23. Feitosa AR, de Ávila AN. Uso do prontuário eletrônico na assistência pré-natal às portadoras de diabetes na gestação. Revista brasileira de ginecologia e obstetrícia. 2016. 38; 9-19.

24. de Azevedo Silveira, AS, de Oliveira CBF, Lessa, F. Prontuário Eletrônico e Gerenciamento de caso em Ambulatório de Psiquiatria. Journal of Health Informatics, 2016: 8(3):83-86.

25. de Anias D, Marinho EF, et al. Aceitação e uso de tecnologias móveis de informação pelos agentes comunitários de saúde de Sapeaçu. Revista Baiana de Saúde Pública. 2017. 41(2).

26. Orfão, NH, et al. Coordenação da assistência à tuberculose: registro de dados e a implementação de um sistema informatizado. Ciência \& Saúde Coletiva. 2017. 22; 1969-1977.

27. Ruschi GEC, et al. Qualidade dos dados de assistência pré-natal na Atenção Básica em prontuário eletrônico e relação com apoio matricial, Vitória, Espírito Santo, 2013-2014: corte transversal. Revista Brasileira de Medicina de Família e Comunidade. 2017. 12(39); 1-13.

28. Cavalcante RB, et al. Informatização da atenção básica à saúde: avanços e desafios. Cogitare Enfermagem. 2018. 23(3).

29. Thum MA, Baldisserotto J, Celeste RK. Utilização do e-SUS AB e fatores associados ao registro de procedimentos e consultas da atenção básica nos municípios brasileiros. Cadernos de Saúde Pública. 2019; 35(2): e00029418.

30. Patrício CM, et al. O prontuário eletrônico do paciente no sistema de saúde brasileiro: uma realidade para os médicos? Scientia Med (Porto Alegre). 2011; 21 (3): 121-31. apud Bacelar S, Almeida WM, Andrade GM. Erros de prontuário. Brasília Med, 2002;39(1/4):42-51.

31. Ruiz LD, Laurino PS, Roma M. Implantação do plano educacional no prontuário eletrônico para pacientes e familiares. Journal of Health Informatics. 2012. 4(1)

32. Cazorla LCM, Gaspar JS, Reis ZSN. Avaliação do impacto da informatização dos registros sobre o parto na produção do conhecimento científico, em um hospital universitário. J. Health Inform. 2016;(8):187193.

33. Colleti J, de Andrade AB, de Carvalho WB. Avaliação do uso de sistemas de prontuário eletrônico nas unidades de terapia intensiva brasileiras. Revista Brasileira de Terapia Intensiva. 2018;(30): 338-46.

34. Pinheiro RS, et al. Cobertura da Comunicação de Informação Hospitalar e Ambulatorial (CIHA) utilizando os partos registrados no Sistema de Informações sobre Nascidos Vivos, Brasil, 2006 a 2009. Cadernos de Saúde Pública. 2012. 28(5); 991-97.

35. Medeiros $\mathrm{K}$ de, et al. Bases de dados orçamentários e qualidade da informação: uma avaliação do Finanças do Brasil (Finbra) e do Sistema de Informações sobre Orçamentos Públicos em Saúde (Siops). Revista de Administração Pública. 2014. 48(5): 1113-33. 